Para enlazar con este artículo / To link to this article:

http://dx.doi.org/10.14198/fem.2019.33.08

Para citar este artículo / To cite this article:

Beorlegui Zarranz, David. «"Detrás de lo que quieren que seamos, está lo que somos". Revolución sexual y políticas sexuales feministas durante las décadas de los setenta y de los ochenta. Una aproximación al caso del País Vasco». En Feminismo/s, 33 (junio 2019): 199-223. DOI: 10.14198/fem.2019.33.08

\title{
«DETRÁS DE LO QUE QUIEREN QUE SEAMOS, ESTÁ LO QUE SOMOS». REVOLUCIÓN SEXUAL Y POLÍTICAS SEXUALES FEMINISTAS DURANTE LAS DÉCADAS DE LOS SETENTA Y DE LOS OCHENTA. UNA APROXIMACIÓN AL CASO DEL PAÍS VASCO
}

\author{
«BEHIND WHAT THEY WANT US TO BE, IS WHAT WE \\ ARE». SEXUAL REVOLUTION AND FEMINIST SEXUAL \\ POLITICS IN THE SEVENTIES AND THE EIGHTIES. \\ AN APPROXIMATION OF THE BASQUE CASE
}

\author{
David BEORLEGUI ZARRANZ \\ Universidad del País Vasco/Euskal Herriko Unibertsitatea \\ dbeorza@yahoo.es \\ orcid.org/0000-0002-8982-5513
}

\section{Resumen}

El objetivo de este artículo es abordar el debate feminista que tuvo lugar en el País Vasco en torno a las políticas sexuales durante las décadas de los setenta y ochenta, al entender que la mirada local se inserta en contextos más amplios y contribuye a una mejor comprensión de los fenómenos históricos. La investigación se basa fundamentalmente en fuentes orales construidas mediante la realización de entrevistas individuales y colectivas a antiguas militantes feministas. Esos testimonios se ponen en relación con fuentes hemerográficas del período y documentación interna producida por distintos colectivos para estudiar el rol específico que se atribuyó a la sexualidad como una parte esencial del activismo feminista. Además de identificar los principales aspectos en los que se basó la política sexual del movimiento, el recorrido histórico propuesto permite apreciar tensiones, debates, cambios y negociaciones en 
«Detrás de lo que quieren que seamos, está lo que somos». Revolución sexual y políticas sexuales feministas durante las décadas de los setenta y de los ochenta. Una aproximación al caso del País Vasco

torno a la sexualidad y su significado en el período histórico comprendidos entre 1975 y 1986.

Palabras clave: Feminismo, sexualidad, lesbianismo, segunda ola, militancia, País Vasco.

\begin{abstract}
The aim of this article is to address the sexual politics feminist debate that took place in the Basque country during the decades of 1970s and 1980s, on the basis that the local perspective is embedded in a broader context and contributes to a better understanding of historical phenomenon. The research is mainly based on oral sources created by recording individual and collective interviews with former feminist activists. These testimonies are related to some periodical press and internal documentation produced by different groups to study the specific role attributed to sexuality as an essential part of feminist activism. In addition to identifying the main aspects in which the movement' sexual policies were based on, the historical view that is proposed provides an insight of the tensions, debates, changes and negotiations around sexuality and its meanings in the historical period between 1975 and 1986.
\end{abstract}

Keywords: Feminism, sexuality, lesbianism, second wave, activism, Basque Country.

\title{
1. INTRODUCCIÓN
}

Parafraseando a Bell Hooks, podríamos afirmar que «el mundo de la sexualidad de las mujeres cambió para siempre con la llegada de la revolución sexual feminista» (Hooks 114). Tal fue el caso, por lo menos, de las mujeres que protagonizaron la «segunda ola» que arrancó a finales de los años sesenta y que se hizo sentir con fuerza en el caso que nos ocupa, el del País Vasco peninsular, coincidiendo cronológicamente con los años de la transición a la democracia. Como recordaba Cristina Garaizábal en las jornadas feministas celebradas en 2009 en la ciudad de Granada, el movimiento se volcó desde los inicios en trabajar el ámbito de la sexualidad, y en centrar buena parte de su crítica en un «modelo de sexualidad dominante» -el masculino heterosexual- que asignaba a las mujeres una posición de inferioridad y supeditación con respecto a los varones (Garaizábal 2). La aportación de las feministas en el terreno de la sexualidad y de los derechos reproductivos es uno de los hechos más destacados de la segunda ola feminista y tuvo, en palabras de

Feminismo/s 33, junio 2019, pp. 199-223 
«Detrás de lo que quieren que seamos, está lo que somos». Revolución sexual y políticas sexuales feministas durante las décadas de los setenta y de los ochenta.

Mary Nash, «un impacto social duradero en la modificación de los valores y de las prácticas públicas y personales en la sociedad» (Nash 199). Pese a la importancia que tuvo la política sexual en la emergencia y el desarrollo del movimiento feminista, su estudio no ha generado una literatura tan amplia como la dedicada a tratar las campañas organizadas por el movimiento en torno a la legalización del divorcio y del aborto (Beorlegui; Epelde, Aranguren y Retolaza; Larumbe; Martínez, Gutiérrez y González; Nash). La originalidad y la influencia de la crítica sexual feminista hacen preciso un análisis de su conformación desde una perspectiva histórica, dado que el significado y las formas que tomó la sexualidad determinaron en buena medida la experiencia de ese movimiento en el contexto de la segunda mitad de los años setenta y el inicio de los ochenta. Sumándonos a la reciente llamada de Inmaculada Blasco para «explorar nuevos territorios y explicaciones en el estudio del feminismo», el texto invita a analizar los significados y contenidos atribuidos a la sexualidad por parte del movimiento, para «explicar mejor cómo se configuraron las identidades y actuaciones que se autodenominaron feministas» (Blasco 282).

Nos proponemos profundizar en torno a la hipotética existencia de una revolución sexual propuesta desde el feminismo en el período comprendido entre 1972 y 1986, así como valorar la incidencia de esas propuestas a la hora de conformar las políticas sexuales del movimiento. Con este propósito analizamos los debates y reflexiones que tuvieron lugar en la militancia feminista del País Vasco, sirviéndonos para ello tanto de documentación generada desde el interior del movimiento como de entrevistas de historia oral realizadas con mujeres que participaron de distintas organizaciones feministas en las provincias de ese territorio. Tomamos como punto de partida la realización de las primeras reuniones y actividades dedicadas al ámbito de lo sexual, por parte de un feminismo todavía embrionario, y concluimos con la existencia de un amplio friso de sensibilidades en el interior de este movimiento. Con ello pretendemos realizar un ejercicio de interpretación que, lejos de ofrecerse como completo, pueda servir como un primer acercamiento para posteriores investigaciones en esta región, u otras que albergaron el desarrollo de grupos análogos en una cronología similar. La primera parte del artículo analiza la influencia de la revolución sexual en el programa emancipador feminista, mientras que la segunda parte explora el significado adquirido

Feminismo/s 33, junio 2019, pp. 199-223 
«Detrás de lo que quieren que seamos, está lo que somos». Revolución sexual y políticas sexuales feministas durante las décadas de los setenta y de los ochenta.

por determinadas prácticas defendidas como irrenunciables por parte del movimiento. La crítica del falocentrismo, el cuestionamiento del coito como práctica de dominación masculina, la exploración de la masturbación y el orgasmo clitoriano, o la reivindicación del lesbianismo, formaron, entre otras cuestiones, una parte fundamental del programa de emancipación feminista durante el período estudiado. La significación de esas prácticas y el peso que ocuparon a la hora de definir la identidad del movimiento experimentó, no obstante, notables variaciones a lo largo del tiempo. El interés del tema en un contexto, el actual, caracterizado por la emergencia de nuevas sensibilidades feministas y de miradas retrospectivas sobre la historia de este movimiento justifica en nuestra opinión sobradamente la realización de este estudio.

La mayoría de las fuentes escritas que se han analizado procede de las organizaciones del movimiento feminista, disponibles en gran medida gracias a la labor de archivo desempeñada por el Centro de Documentación de las Mujeres Maite Albiz ${ }^{1}$. Contamos así con información procedente de los dosieres editados por la Asamblea de Mujeres tras las Jornadas de la Mujer de Leioa, celebrada en diciembre de 1977, así como el dosier «Sexualitate eta amatasun askatu baten alde/Por una sexualidad y maternidad libres», editado en 1981 por la Comisión de la mujer de Algorta, integrada dentro de la asociación vecinal «Askatasuna». A ello se suman los materiales editados tras el I Encuentro de Lesbianas de Euskadi, celebrado los días 21 y 22 de mayo de 1983 en la localidad guipuzcoana de Errentería, y los pertenecientes a las II Jornadas de Lesbianas Feministas de Euskadi, celebradas del 1 al 3 de mayo de 1987 en Orio. También hemos incluido, por último, distintos textos publicados durante los años setenta y ochenta en publicaciones periódicas como El País, Egin o Zer Egin, así como una entrevista recogida en el primer número de la revista Sorginak, realizada en 1986. La lectura de estas fuentes nos ha permitido reconstruir buena parte de los intensos debates que tuvieron lugar en el seno del movimiento, tanto en sus encuentros como en

1. Situado en la ciudad de Bilbao, el Centro lleva más de dos décadas recogiendo documentación alusiva al mundo de las mujeres, contando además con un amplio fondo dedicado al movimiento feminista. Buena parte de esa documentación se encuentra además digitalizada y accesible a través de Internet.

Feminismo/s 33, junio 2019, pp. 199-223 
«Detrás de lo que quieren que seamos, está lo que somos». Revolución sexual y políticas sexuales feministas durante las décadas de los setenta y de los ochenta. Una aproximación al caso del País Vasco

la prensa, y apreciar la importancia que se le asignaba a todo lo relacionado con la sexualidad.

La realización de entrevistas de historia oral constituye una vía privilegiada para la investigación del pasado siglo veinte. Analizadas desde las premisas de una historia oral de tipo interpretativo, las fuentes orales desempeñan un papel importante en la conformación del estudio. Las entrevistas estudiadas tienen distintos orígenes y han sido realizadas entre los años 2009 y 2017. La mayor parte de los testimonios pertenecen a las colecciones «Luchas obreras en Bizkaia» y «Activismo sociopolítico en el País Vasco las décadas de los setenta y los ochenta», realizadas respectivamente por Mentxu Irusta Laforga y David Beorlegui y depositadas en el Archivo de la Memoria (AHOA) del País Vasco. Se trata en todos los casos de historias de vida con personas que participaron de distintos movimientos políticos, sociales y sindicales contando además con varias entrevistas a antiguas militantes feministas. La mayoría de las narradoras nacieron a finales de los años cuarenta e inicios de los cincuenta, formando parte de la generación conocida como el «baby boom», criada en los años del desarrollismo franquista. Vinculadas en su gran mayoría a distintas formaciones de la izquierda radical (MC-EMK, LCR-LKI, OIC, EE), todas ellas impulsaron el movimiento feminista a partir de la creación de grupos de mujeres en sus respectivos barrios y pueblos, desarrollando su actividad en las localidades de Getxo, Bilbao, Barakaldo, Rentería y Donostia. También contamos con dos entrevistas colectivas que, a modo de grupos de rememoración, se realizaron por el último autor en el año 2017 con una docena de mujeres que impulsaron la creación de colectivos feministas en los barrios de Algorta y Romo, situados en Getxo (Bizkaia). Pese a que por razones de tiempo la muestra se limita a mujeres procedentes de los territorios de Gipuzkoa y Bizkaia, consideramos que se trata de un elenco de voces lo suficientemente representativo como para realizar un acercamiento preliminar a la cuestión de la sexualidad durante la segunda ola feminista en el País Vasco. El cómputo global de entrevistas trabajadas para este texto asciende a ocho.

Desde la dimensión subjetiva que caracteriza siempre a la historia oral, en lo que constituye su principal fortaleza, los relatos vitales de las militantes feministas son muy prolíficos en información relativa a su participación en los distintos organismos de oposición al régimen, pudiendo además reproducir

Feminismo/s 33, junio 2019, pp. 199-223 
«Detrás de lo que quieren que seamos, está lo que somos». Revolución sexual y políticas sexuales feministas durante las décadas de los setenta y de los ochenta.

con una gran cantidad de detalle las tensiones de género existentes en esas organizaciones. También constituyen un testimonio privilegiado para analizar la subjetividad feminista, esto es, el modo mediante el que las mujeres se hicieron feministas e hicieron del feminismo un elemento constitutivo de su relación con el mundo. La información y los recuerdos que refieren a la sexualidad, en cambio, son más escasos y se centran fundamentalmente en los aspectos más técnicos de la misma, esto es, las cuestiones vinculadas con la natalidad o las campañas que se realizaron en torno a la legalización de los anticonceptivos, el aborto, o la denuncia de la violencia sexual. Los testimonios son todavía más exiguos en lo que respecta a las prácticas sexuales, que parecen mantenerse limitadas a los terrenos de lo íntimo y de lo privado, considerándose información inapropiada para comentar en el transcurso de una entrevista. Esta es una cuestión del todo comprensible, aunque un tanto paradójica, sobre todo si se atiende al hecho nada baladí de que los asuntos íntimos, personales, propios de la vida privada, resultaron fundamentales para teorizar en torno a la opresión de las mujeres y para vertebrar el movimiento feminista, pasando a formar parte irrenunciable de su programa de transformación personal y colectiva.

Los tabús que existen en torno al sexo no son los únicos aspectos problemáticos de la historia de la sexualidad. Otra de las dificultades tiene que ver con la vigencia de los planteamientos foucaultianos, tendentes a concebir la sexualidad como un campo regido por lógicas eminentemente disciplinarias. La tarea de abordar el tema desde una perspectiva que mire más allá de los límites impuestos por el lenguaje es todavía más complicada por el hecho de que la mayoría de fuentes de las que disponemos se limitan a informar sobre lo que determinados grupos y sectores sociales decían en torno al sexo, más que sobre las prácticas sexuales, la conciencia sexual, o el placer sexual en sí. Sin despegarnos del todo del análisis discursivo, las fuentes orales nos permiten ensanchar nuestra comprensión de la sexualidad en el ámbito del feminismo, al brindarnos la posibilidad de explorar los modos mediante los que las mujeres aprehendieron, experimentaron, discutieron, asimilaron o rechazaron las políticas sexuales configuradas desde el movimiento, así como la manera mediante la que esas premisas son recordadas, interpretadas, trasmitidas y expresadas en el presente. Ello incluye tanto los términos mediante los que

Feminismo/s 33, junio 2019, pp. 199-223 
«Detrás de lo que quieren que seamos, está lo que somos». Revolución sexual y políticas sexuales feministas durante las décadas de los setenta y de los ochenta. Una aproximación al caso del País Vasco

se dio significado a la subjetividad feminista como las emociones que la acompañaron y dieron forma en tanto que experiencia individual y colectiva.

\section{LA REVOLUCIÓN SEXUAL DE LA SEGUNDA OLA FEMINISTA (1977-1981)}

En su breve ensayo sobre la historia del feminismo en el País Vasco, Begoña Zabala citaba a Justa Montero para afirmar que «el trabajo, la familia y la sexualidad» fueron los tres «grandes epígrafes» que estructuraron desde los inicios buena parte de la propuesta feminista (Zabala 2008). Pese a la importancia que tuvo la cuestión sexual a lo largo de los años setenta y ochenta, los trabajos dedicados al estudio del feminismo han otorgado una prioridad en el análisis a las movilizaciones que tuvieron lugar en torno a objetivos concretos. Ello tiene como resultado una cierta tendencia a subsumir las lógicas del movimiento en la estructura de oportunidad política que se va generando en el transcurso de la transición. Aunque la mayoría de trabajos no han reparado en la centralidad que tuvo la cuestión sexual en la conformación del movimiento, hay algunas aproximaciones que aprecian la importancia de ese factor en el desarrollo del movimiento de las mujeres en el territorio vasco. Nos referimos en concreto a una reflexión de carácter semiautobiográfico presentada por Cristina Garaizábal en las Jornadas Feministas de Granada de 2009. Esta veterana militante y psicóloga feminista planteaba que la «crítica a la hegemonía masculina en las relaciones sexuales, acompañada de la defensa del derecho al placer sexual para las mujeres constituyeron los ejes fundamentales de la visión feminista de la sexualidad en los primeros años de nuestro movimiento feminista» (Garaizábal 1-2).

Revisando las obras feministas que formaban parte de su biblioteca personal, Mary Nash reparaba en la importancia que habían tenido las cuestiones relacionadas con el cuerpo y la sexualidad en el desarrollo histórico del movimiento. Para esta historiadora y antigua militante, el movimiento de liberación de las mujeres consiguió colocar la sexualidad de las mujeres en el centro de los debates políticos, rompiendo toda una serie de tabús históricos y tomando posiciones colectivas frente a uno de los procesos culturales más relevantes de los años sesenta, la revolución sexual. Las feministas partieron de una crítica a la sexualidad vigente, interpretando a esta como una extensión

Feminismo/s 33, junio 2019, pp. 199-223 
«Detrás de lo que quieren que seamos, está lo que somos». Revolución sexual y políticas sexuales feministas durante las décadas de los setenta y de los ochenta.

de una masculinidad dominante, como un gesto narcisista de los hombres que desproveía a las mujeres de entidad y derecho al placer propio. La publicación en 1967 del influyente estudio Masters-Johnson ya había dejado constancia de la enorme diversidad y potencialidad de la sexualidad de las mujeres, rompiendo muchos de los estereotipos que existían entonces sobre la frigidez, procedentes del discurso médico, y reivindicando el orgasmo clitoriano frente al vaginal ${ }^{2}$. Igual sucedía con las obras de Simone de Beauvoir, Kate Millet, Shulamith Firestone o Carla Lonzi, de cuya lectura tenemos constancia para el caso de los grupos de Bizkaia. Trabajos como «El segundo sexo», «La dialéctica del sexo», «Sexual politics»o «Escupamos sobre Hegel» permitieron a las feministas cimentar teóricamente sus políticas sexuales (Nash 178-180).

La aparición de corrientes de nueva izquierda y la influencia de una contracultura difusa en el mundo de la oposición antifranquista implicaron cambios importantes en los modelos de la militancia, así como la recuperación de algunas obras que, como sucedía con los trabajos de Wilhelm Reich, por ejemplo, habían pasado inadvertidos hasta el momento y subrayaban la necesidad de llevar a cabo políticas sexuales en un sentido emancipador. Procedentes en su mayoría de ese ámbito de la oposición clandestina, las entrevistadas recordaban que, de modo todavía muy tímido y semioculto, la sexualidad había estado presente desde los primeros momentos de su politización más temprana. Estíbaliz Beitia recuerda como en torno al año 197273, distintos partidos ofrecían charlas sobre: «la planificación familiar, (el) aborto (la) sexualidad», recogidas bajo el calificativo de «cursos de medicina cubana». «Antes la medicina cubana era muy oída -aseguraba- Entonces... nos daban charlas a los de EIA ${ }^{3}$, para poder plantear la planificación, de forma preventiva. Entonces estabas en eso, con gente de la ORT... con un pirao, que daba los cursos esos» ${ }^{4}$. El movimiento vecinal fue otro de los espacios en los que las feministas comenzaron a ofrecer cursos de sexualidad, como sucedía, por ejemplo, en la Universidad Libre de Rekaldeberri, una institución surgida

2. Los resultados de ese estudio, como advierte Nash, llegaron a ser divulgados por Vindicación Feminista y tuvieron una influencia más notable para el caso español.

3. Euskal Iraultzarako Alderdia (Partido para la Revolución Vasca).

4. Entrevista a Estíbaliz Beitia. Nacida en 1956 en Erandio, Bizkaia. Formó parte de EIA durante los años sesenta y de la Comisión de la Mujer de Romo, así como de la Asamblea de Mujeres de Bizkaia.

Feminismo/s 33, junio 2019, pp. 199-223 
«Detrás de lo que quieren que seamos, está lo que somos». Revolución sexual y políticas sexuales feministas durante las décadas de los setenta y de los ochenta.

en un barrio bilbaíno que contaba con un módulo de formación dirigido a las mujeres. María Luisa Menéndez recuerda que en aquellos talleres: «Era todo voluntario [...], organizar debates o cosas, y dar... a todas las mujeres que venían a por anticonceptivos, previamente unas charlas sobre sexualidad $»^{5}$.

En opinión de la antropóloga Mari Luz Esteban, este tipo de planteamientos colectivos que giraban en torno al cuerpo y la sexualidad de las mujeres durante los años setenta se constituyeron como una «palanca primordial» para la creación de «cuerpos políticos» feministas (Esteban 81). Evocando su participación en el grupo de mujeres de Romo, en la localidad de Getxo, Marisol Fuertes recuerda haber asistido a charlas sobre sexualidad, relacionadas con temas como «la planificación familiar», «los anticonceptivos», o el «conocer nuestro propio cuerpo». Ella recuerda que la gente que acudía a esos eventos «participaba muchísimo, participábamos bastante. No nos daba vergüenza, decíamos lo que nos pasaba, lo que nos dejaba de pasar. Había gente diferente...». Durante el tiempo que duraba la discusión, reitera, esta transcurría en términos de los más animados: «no callábamos, ¿eh?, hablábamos de muchas cosas, no teníamos problemas en hablar de cualquier cosa ${ }^{6}$. Otra de las participantes en esos talleres, Miren Isasi, señala que el sexo había sido para su generación «un tabú tan tremendo, y más para las mujeres, si las mujeres se interesaban, eran putas, pues supongo... y teníamos tal desconocimiento y tal desconcierto, hablábamos mucho». Hablar de sexo, relata esta veterana militante feminista, «era... nuestro, pero era más... político, también. Y la verdad es que nos soltamos bastante» ${ }^{7}$. Lejos de disminuir con los inicios de la transición política, la relación entre el sexo y la política se vería incrementada hasta pasar a ser un tema insignia en los debates impulsados por parte del movimiento feminista.

Surgidos al calor de la efervescencia reivindicativa de la transición, los grupos de autoconciencia que proliferaron durante la segunda mitad de los

5. Entrevista a María Luisa Menéndez. Nacida en 1950 en Bilbao. Formó parte de ASK y EE, pasando posteriormente al sindicato LAB. Desde los años setenta hasta la actualidad ha colaborado con la Asamblea de Mujeres de Bizkaia.

6. Entrevista a Marisol Fuertes. Fecha y lugar de nacimiento desconocidos. Formó parte del MC-EMK, de la Comisión de la Mujer de Romo y de la Asamblea de Mujeres de Bizkaia.

7. Entrevista a Miren Isasi. Nació en Bilbao en 1950. Formó parte de la Comisión de la Mujer de Algorta y de la Asamblea de Mujeres de Bizkaia.

Feminismo/s 33, junio 2019, pp. 199-223 
«Detrás de lo que quieren que seamos, está lo que somos». Revolución sexual y políticas sexuales feministas durante las décadas de los setenta y de los ochenta.

años setenta no iban a pasar la oportunidad de incluir sus demandas y presionar en pos de acometer cambios profundos que transformaran sustancialmente la vida de las mujeres, insistiendo una y otra vez en la necesidad de acometer una revolución sexual feminista. Las jornadas feministas fueron fundamentales para presentarse a otras mujeres y manifestar la fuerza del movimiento. En el caso del País Vasco las primeras Jornadas de la Mujer se organizaron en diciembre de 1977 en la localidad de Leioa, invitando a las asistentes a sumarse a una larga carrera por construir un modelo distinto de relaciones humanas: "Cuando hablamos de sexualidad iqué queremos? -planteaban durante esas jornadas- queremos denunciar los mitos que nos han destruido. Queremos salirnos de ellos y solidarizarnos entre las mujeres para llegar a unas relaciones amorosas y abiertas, pero antes de amar a otros es necesario amarse uno mismo, respetar nuestras necesidades y mirar nuestra sexualidad de frente» (Jornadas de la Mujer de Euskadi, II: 9). Las dudas y sospechas que, heredadas de los planteamientos contraculturales, se cernían sobre la sexualidad, dieron paso a una búsqueda acelerada de una sexualidad y de una experiencia auténticamente liberadoras y feministas, descritas en términos como «expresar de alguna forma nuestro deseo, saber cuál es, llegar a situar lo que sentimos, liberar nuestra sensualidad, dejarnos llevar por todas las sensaciones, ver, oír, tocar, que también nos llenan de placer». "¿Y cuáles son las fuentes de nuestro deseo? -referían las activistas- ¿Solamente el hombre? Pues nosotras no lo creemos» (Jornadas de la Mujer de Euskadi, I: 15).

Las feministas vascas consideraban que la revolución sexual era una tarea todavía pendiente a finales de los años setenta, argumentando que: «entendida como desarrollo de las posibilidades de comunicación y placer de la persona humana», la sexualidad continuaba siendo patrimonio exclusivo de los varones. «Mientras que se considera normal y justo el que los hombres tengan una vida sexual activa y satisfactoria [...] se limita la sexualidad femenina al matrimonio, orientándola única y exclusivamente hacia la procreación». Las mujeres que conformaban el movimiento entendían que su sexualidad se desplegaba «en un medio hostil lleno de miedos e inhibiciones», haciendo precisa la elaboración de un programa de intervención sexual dirigido a emancipar a las mujeres de esa carga, dadas las dificultades que existían para pensar la sexualidad «al margen de éxitos y fracasos», planteando que «ni

Feminismo/s 33, junio 2019, pp. 199-223 
«Detrás de lo que quieren que seamos, está lo que somos». Revolución sexual y políticas sexuales feministas durante las décadas de los setenta y de los ochenta.

una sexualidad limitada a la procreación, ni una sexualidad manipulada por el consumo liberan a la mujer [...] frente a esto defendemos el derecho de la mujer a desarrollar una sexualidad que no se vea limitada a la procreación; el derecho a decidir libremente las relaciones sexuales...»(Jornadas de la Mujer de Eusi, I, 36).

La ruptura con el orden establecido pasaba por acometer una revolución sexual capaz de trascender la moral represiva heredada del nacional-catolicismo y el paradigma supuestamente liberal o propio capitalismo avanzado, tendente a identificar a la mujer con un objeto de consumo. Se trataba por tanto de construir una cultura a la contra de los valores dominantes, que hacían del cuerpo de las mujeres el principal campo de batalla de las tensiones entre lo viejo y lo nuevo. Se trataba, en otras palabras, de dotar de significado a la revolución sexual, de no ahogar las posibilidades de emancipación en la liberación de las costumbres representada, entre otros, por fenómenos como el «destape». Tal y como podía leerse en una entrevistada realizada con las promotoras de los encuentros, estas entendían que la sexualidad de las mujeres estaba atrapada: «entre dos muros: el represivo, que nos identifica a objetos reproductores, y el mal llamado liberador, que nos identifica a objetos de placer». En un contexto, el de la transición, caracterizado por acelerados cambios políticos y culturales, las feministas mostraron gran interés en que sus opiniones sobre la sexualidad fueran tenidas en cuenta, denunciando que: «lo mismo que en el resto de los apartados de la Constitución [...] nadie ha preguntado lo que opinamos ni vamos a tener oportunidad de decidir lo que queremos». Primero en grupos pequeños, luego en campañas más amplias, las activistas animaban a las mujeres a sumarse a las movilizaciones que existían «en Euskadi y en todo el Estado español, campañas por una sexualidad libre, donde todas podamos participar y buscar una información más de acuerdo con nuestros deseos y necesidades reales» («Sexualitate eta amatasun»1).

Una de las principales preocupaciones de las mujeres tenía que ver con el hecho de que la sexualidad «no solo ha estado reprimida, oculta y prohibida, sino que ha estado y está englobada en la función maternal» («Sexualitate eta amatasun» 2). El objetivo inmediato de la labor de las activistas, por tanto, era desvincular la sexualidad de las mujeres de la maternidad, así como concienciar a las mujeres de la importancia de controlar su natalidad mediante el uso de anticonceptivos. La responsabilidad de no contraer un embarazo,

Feminismo/s 33, junio 2019, pp. 199-223 
«Detrás de lo que quieren que seamos, está lo que somos». Revolución sexual y políticas sexuales feministas durante las décadas de los setenta y de los ochenta.

por tanto, seguía recayendo fundamentalmente en las espaldas de las mujeres, siendo este un hecho patente en opinión de las feministas de finales de los años setenta: «solo una de las dos partes parece que está interesada en tomar las medidas necesarias para que no siempre que se coite vengan niños», denunciaban. La consecuencia inmediata de esa situación, en el intervalo entre los años setenta y ochenta, era la de asignar una prioridad absoluta a la legalización de los anticonceptivos, «porque la penetración del pene en la vagina parece ser el sinónimo del amor sexual en esta sociedad» («Sexualitate eta amatasun» 8). Abogando por cuestionar «ese viejo estereotipo e intentar que las relaciones sexuales no tengan, obligatoriamente, que pasar por un solo modelo universal», la propuesta del feminismo buscaba explorar caminos menos determinados «por la genitalidad, por la penetración, puesto que por el simple frote el hombre puede eyacular rápidamente, cuando se puede afirmar que las mujeres necesitamos expresarnos eróticamente con mucho más que penes y vaginas» («Sexualitate eta amatasun» 2 ). Todo ese esfuerzo por desarrollar una sexualidad que se desarrollara en cauces distintos a la cultura dominante, masculina y heterosexual, invistió a la revolución sexual feminista de un contenido utópico que no había tenido lugar en la incorporación, en clave liberal, de la «liberación sexual», limitada a una cierta relajación de las costumbres y un aumento del acceso masculino al cuerpo de las mujeres.

El impulso de articular una propuesta alternativa a la sexualidad dominante subyacía en aseveraciones como las contenidas en la revista Geu Emakumeok en el año 1985, permitiendo apreciar lo que algunas autoras como Gayle Rubin han denominado «feminismo pro-sex», basado en la crítica de «una ortodoxia sexual en el nombre del feminismo», abogando «por la defensa de la inocencia sexual hasta que la culpa fuera probada, y no al revés» (Rubin 267-320; Willis XIX). Negando todo acercamiento al sexo que pudiera interpretarse como moralista o puritano, las activistas manifestaban de modo explícito que: «la reivindicación del placer debe formar parte de los objetivos que perseguimos como feministas», manifestando su voluntad de «luchar por una sexualidad diferente, en la que todo nuestro cuerpo participe», afirmando creer firmemente en «la magia de lo imprevisto». Entre los objetivos marcados, enumeraban el «romper con lo establecido, con los roles tradicionales, con la heterosexualidad como único modelo, con el número dos como única forma de relación posible». Manifestando la dimensión militante

Feminismo/s 33, junio 2019, pp. 199-223 
«Detrás de lo que quieren que seamos, está lo que somos». Revolución sexual y políticas sexuales feministas durante las décadas de los setenta y de los ochenta.

y utópica de su lucha por la revolución sexual, las mujeres concluían el texto con un llamamiento a realizar «una lucha política que subvierte los modelos culturales que se nos imponen, y abre un camino hacia una nueva sociedad, en la que el amor sea manifestación de vida y de alegría y no de absurdas entregas» («Sobre el amor romántico y la sexualidad» 2),

Entre finales de los años setenta e inicio de los ochenta el movimiento feminista tomó la decisión de intervenir en el campo de la sexualidad, al entender que era uno de los principales elementos de discriminación y violencia contra las mujeres. Una vez obtenido el diagnóstico, la sexualidad pasó a ocupar un primer plano en la agenda política de las feministas, junto a la presión por acometer reformas legales que pusieran fin a los llamados «delitos femeninos» (adulterio, divorcio, aborto...) y a la situación de discriminación que experimentaba, al menos la mitad de la población. La sospecha que se introdujo sobre el campo de la sexualidad por parte de las activistas dotó a esta de un significado político que, sin dejar de lado su dimensión más utópica, pronto se materializó en toda una serie de cuestiones concretas a incorporar en la vida diaria de las militantes, de prácticas afectivas vinculadas a la conformación de nuevas subjetividades, que se convirtieron en verdaderas consignas del movimiento. La introducción de esos planteamientos y la experimentación con nuevas maneras de amar y sentir fueron aspectos fundamentales para el desarrollo del feminismo histórico y quedaron recogidos tanto por la documentación manejada por los distintos grupos como por las memorias de quienes participaron en ellos. En el siguiente punto, pasamos a comentar algunas de las propuestas extraídas de los programas reivindicativos feministas que implicaban la adopción de prácticas sexuales determinadas.

\section{LAS POLÍTICAS SEXUALES DEL MOVIMIENTO FEMINISTA (1981-} 1987)

Para comienzos de los años ochenta las feministas tenían un diagnóstico claro en lo que refería a su vida sexual: «sexualmente, somos lo que los hombres quieren que seamos, como en todo lo demás [...] pocas mujeres sentimos, aunque tenemos orgasmos, que es esto exactamente lo que queremos» («Sexualitate eta amatasun»4). Esa insatisfacción que las activistas hacían extensiva al conjunto de las mujeres se relacionaba a su juicio con «un

Feminismo/s 33, junio 2019, pp. 199-223 
«Detrás de lo que quieren que seamos, está lo que somos». Revolución sexual y políticas sexuales feministas durante las décadas de los setenta y de los ochenta.

problema de fondo, que tiene que ver más con la sexualidad que con la anticoncepción misma», lo que requería abandonar algunas prácticas y plantear alternativas a una sexualidad, la dominante, que consideraban alienante y opresiva. La elaboración de una subjetividad feminista iba a dar lugar en ese sentido a nuevos acercamientos discursivos a la cuestión sexual, materializados en toda una serie de prácticas y disposiciones que favorecieron a su vez el surgimiento de nuevas posibilidades y experiencias. Entre las prácticas a adoptar como parte de un proyecto más amplio de transformación política, destacan la crítica a la penetración, la reivindicación del orgasmo femenino y del clítoris, y la asunción del lesbianismo.

Tomando como válida la síntesis que ofrece Garaizábal en su texto autobiográfico, los esfuerzos del movimiento se volcaron hasta comienzos de los años ochenta en cuestionar: «que la penetración vaginal fuera el modelo para las relaciones sexuales entre hombres y mujeres; igualmente planteamos que la vagina no tenía por qué ser el órgano sexual por excelencia de las mujeres y empezamos a reivindicar el papel que jugaba el clítoris en el placer sexual femenino» (Garaizábal 1-2). La novedad que representaban esos planteamientos para las mujeres de los años setenta queda reflejada de modo muy expresivo en el relato de Mariví Marañón, que convierte su asistencia a los encuentros de Leioa en un enclave de su memoria (Llona 19; 50-51) en el sentido de conectar con el presente por medio de las emociones que invoca su recuerdo de lo experimentado entonces: «Las Jornadas de Leioa, en el setenta y ocho (sic), fue para mí... < ¿qué es esto?> Esa impresión de que una cosa tan amplia, tan gorda, tan... todo lo dirigen las mujeres, lo hacen las mujeres. Entonces... eso fue para mí un flash. Luego, que si el lesbianismo, que si no sé qué... ¡buah!, fue una cosa... de ojos de plato saliendo de ahí» ${ }^{8}$.

Cuando sometemos estos textos al análisis discursivo, es preciso advertir, como lo hace Nash, que la mayoría de las mujeres de la época tenían conocimientos más bien rudimentarios en torno al funcionamiento de su sexualidad dado que «los patrones culturales tradicionales de la sexualidad femenina habían conllevado una mutilación simbólica de las mujeres, al negar

8. Entrevista a Mariví Marañón. Nacida en 1943 en Medina de Pomar (Burgos), formó parte de la JOC, el MC-EMK, y de la Asamblea de Mujeres de Bizkaia. En la actualidad sigue militando en el movimiento feminista.

Feminismo/s 33, junio 2019, pp. 199-223 
«Detrás de lo que quieren que seamos, está lo que somos». Revolución sexual y políticas sexuales feministas durante las décadas de los setenta y de los ochenta.

su sexualidad y anestesiar cualquier expresión de placer sexual femenino, por considerarse antinatural y pecaminosa». La publicidad del orgasmo femenino, en ese sentido, supuso en palabras de esta autora «una ruptura extraordinaria de la práctica sexual», que sintetiza en una pancarta exhibida en 1972 por un grupo de feministas anglosajonas: «Think Clitoris» («Piensa clítoris») (Nash 199-200). El conocimiento del propio cuerpo pasaba por ser condición ineludible para la constitución de la nueva subjetividad feminista, poniendo como ejemplo que: «las mujeres podemos tener orgasmos siempre y cuando se nos ocurra, a condición de que nuestro clítoris sea estimulado directa o indirectamente. Y podemos tener varios orgasmos continuados o separados en un breve espacio de tiempo». Una vez constatado ese hecho, la conclusión de las activistas no dejaba lugar a equívocos: «si las mujeres alcanzan el orgasmo a través de la masturbación y no de la penetración, lo que hay que cuestionar es el valor de la penetración y no al revés» («Sexualitate eta amatasun» 5).

Además de apoyarse en razonamientos higiénicos, políticos y prácticos en su crítica al coito, las feministas también añadían argumentos de tipo cuantitativo que revelan la influencia de los trabajos de Kinsey, Master y Johnson, Koed o Hite, planteando que: «si nunca has tenido un orgasmo, y quieres tenerlo, puedes intentar masturbarte, en un 95\% de los casos, se accede con facilidad al orgasmo y solamente en un 30\% de las mujeres se consigue a través de la penetración» («Sexualitate eta amatasun» 5). El orgasmo femenino aparecía como uno de los elementos definitorios de la sexualidad de las mujeres y se definía como «un alivio repentino o involuntario y casi instantáneo de la congestión pélvica y la tensión muscular, acompañado por contracciones musculares», asociado a un «enorme placer». La vagina, por contra, aparecía descrita como «el sitio por donde sale el flujo, la menstruación y los niños. Sitio por donde entran el espéculo, un tampón o un pene. No tiene terminaciones nerviosas importantes. Es casi insensible. Puede sentir orgasmos, pero estos ocurren en el clítoris». Unas líneas más adelante, el dosier editado por las mujeres del barrio getxotarra volvía a insistir en que «frote, caricia con la mano, la lengua o con cualquier otra cosa [...] de los pezones o cualquier otra zona erógena, o de las emociones, el orgasmo ocurre siempre en el clítoris. Esto significa que una mujer no es frígida si no tiene orgasmos cuando su clítoris no está excitado suficientemente, como ocurre con una penetración apresurada, sin estimulación previa» («Sexualitate eta amatasun»

Feminismo/s 33, junio 2019, pp. 199-223 
«Detrás de lo que quieren que seamos, está lo que somos». Revolución sexual y políticas sexuales feministas durante las décadas de los setenta y de los ochenta.

5). La labor de estos grupos en lo que respecta al «descubrimiento» y la politización del placer sexual, así como el impacto de sus propuestas, fue sin lugar a dudas enorme. El hincapié en los aspectos más materiales de la sexualidad, como el cuerpo o la conducta, por otra parte, implicó en opinión de Cristina Garaizábal un cierto descuido de la dimensión simbólica de las relaciones sexuales, como pueden ser las relativas a los deseos y las fantasías eróticas (Garaizábal 2).

En los textos que distribuían entre las mujeres, las feministas planteaban que «todo acto sexual en el que no has conseguido expresar y gozar todo lo que querías, algo en ti ha sido violado, tu propia concepción de la sexualidad, aunque ni te atrevas a confesártelo a ti misma, aunque no te haya sido permitido conocer». La penetración no sólo se asociaba al riesgo de embarazos no deseados, sino también al «contagio de enfermedades venéreas, la frustración sexual común cuando el hombre eyacula y no hemos llegado al orgasmo, la frustración sexual común de saber que no llegaremos al orgasmo...» («Sexualitate eta amatasun» 8-9). Atendiendo a esas cuestiones de tipo fisiológico y político, las feministas terminaban por incluir en el texto toda una serie de recomendaciones prácticas, planteando, por ejemplo, que: «la posición tradicional del coito no favorezca la estimulación del clítoris en algunas mujeres. Si este es tu caso, prueba otras posiciones la próxima vez hasta que encuentres la buena. O no aceptes la penetración. Hay otras formas tanto para la mujer como para el hombre de tener una relación sexual plenamente satisfactoria» («Sexualitate eta amatasun» 4-5). Esa negativa a realizar el coito fue un aspecto central de las políticas sexuales feministas a finales de los años setenta. La consideración de esta práctica era en general muy negativa, en tanto que se concebía fundamentalmente como una agresión a las mujeres, muestra de la dominación y el poder masculinos. «afirmación del precepto primero de la virilidad, que dice: penetra, posee, somete al inferior que tienes debajo y siéntete alguien» («Sexualitate eta amatasun» 2-3). En términos análogos a los expuestos por el dosier de Algorta, las integrantes de la de la Asamblea de Mujeres de Bizkaia definían la penetración como «el acto sexual a través del cual se considera que el hombre posee a la mujer y esta se entrega» (Comisión antiagresión de la AMB 16).

Edurne Eraso guarda un recuerdo ambivalente de su participación en el feminismo pese a considerar que este constituyó «un espacio natural para

Feminismo/s 33, junio 2019, pp. 199-223 
«Detrás de lo que quieren que seamos, está lo que somos». Revolución sexual y políticas sexuales feministas durante las décadas de los setenta y de los ochenta.

mí». Esa sensación guarda relación, en opinión de esta guipuzcoana, con el hecho de «quizás haber tomado demasiado al pie de la letra» sus políticas sexuales. Como recuerda esta entrevistada, el rechazo a tener coitos tenía su origen en el hecho de que: «los hombres solo querían follar mediante la penetración. Entonces, realmente, era una práctica política decir: «¡No, yo no quiero esto, joder!», es que no lo querías, decías: «vamos a hacer otras cosas, ¿no?». Con un cierto distanciamiento irónico con respecto a la adopción de esas prácticas, ella refiere que: «hubo un tiempo en el que... joder, en el que la penetración era un pecado mortal. Claro, porque los hombres eran agresores... entonces, en ese sentido, estabas cortando una práctica que también era posible» ${ }^{9}$. En un proceso de conformación de un sujeto político en torno a parámetros compartidos y a una identidad sólida y afirmada en principios binarios, no había lugar para posturas que dieran lugar a la más mínima sospecha de complicidad con el heterosexismo, de modo similar a lo acontecido en otras latitudes del globo (Willis 28-29). En un tono igualmente crítico con respecto a la adopción de esa práctica política como consigna del movimiento, la activista Paloma Uría reflexionaba en una obra publicada en 2009 sobre las políticas sexuales del movimiento durante las décadas de los setenta y ochenta, al entender que estas contribuyeron, por una parte, a «acentuar la tendencia normalizadora inherente al feminismo de la época, y, por otra, dejó desarmadas a las mujeres, que en voz baja, de forma vergonzante, confesaban que ellas, a pesar de los pesares, sentían placer con el coito» (Uría 102).

Pese a la existencia de discrepancias en el seno del movimiento por las políticas sexuales que emanaban de su interior, las feministas eran conscientes de que una buena parte de su programa utópico estaba basado en «tratar de redescubrir otra sexualidad donde todo nuestro cuerpo participe, donde exploramos en nosotras y en todas las zonas erógenas apenas utilizadas para el placer, donde el clítoris tenga la principal atención, donde el pene no necesita solamente un agujero y descubra otras posibilidades de satisfacción». Se trataba, en definitiva, de un terreno «donde el juego sexual, el intercambio

9. Entrevista a Edurne Eraso. Nacida en el Goierri, Guipúzcoa, en 1960. Formó parte de la Asamblea de Mujeres de Bizkaia, de grupos de lesbianas feministas y de casas «okupadas» de mujeres durante los años ochenta y noventa. En la actualidad forma parte de un grupo de mujeres en Bizkaia.

Feminismo/s 33, junio 2019, pp. 199-223 
«Detrás de lo que quieren que seamos, está lo que somos». Revolución sexual y políticas sexuales feministas durante las décadas de los setenta y de los ochenta.

amoroso, las caricias, sean primordiales», donde el sexo pasaba por expresarse «en mil relaciones cuerpo a cuerpo diferentes, que no incluyan necesariamente la eyaculación dentro de la vagina» («Sexualitate eta amatasun»14). En términos muy similares a los manejados por sus compañeras vizcaínas, las mujeres del grupo autónomo de Guipúzcoa también entendían el feminismo como un impulso por «la consecución de una auténtica liberación en una sociedad en donde el placer sensual no esté definido en función del goce del hombre ni deba ir necesariamente unido a la procreación», mostrándose partidarias de potenciar «la sensualidad, la sensibilidad y el afecto» (Angulo). La ansiedad por experimentar con una sexualidad radicalmente distinta a la que existía hasta el momento también quedaría reflejada años más tarde en las páginas de Geu Emakumeok, el principal órgano de expresión del movimiento, al plantear que «el rollo es buscar, buscar lo que nos han negado que tenemos y buscar lo prohibido, lo oculto, lo que no nos atrevemos a pensar [...] detrás del miedo, está el placer. Detrás de lo que quieren que seamos, está lo que somos» (Mujeres jóvenes de Institutos, FP Y Facultades, «Basta» 3).

Las feministas se habían presentado a la sociedad con la voluntad de poner nombre a lo que hasta entonces había permanecido oculto y encerrado. Y no había realidad más oculta que la de las mujeres lesbianas. Así, algunas de ellas habían denunciado durante los encuentros de 1977 que: «ni siquiera tenemos derecho de existir. Las lesbianas no existimos. [...] la cárcel es la calle y la cárcel, también eres tú mujer, que cuando hablas de sexualidad hablas de coitos, penetración...» (Jornadas de la Mujer de Euskadi, I: 16). Isabel García recuerda que en esos momentos conoció «a unas mujeres que se juntaban. Y ahí entré. Era específico de un movimiento de lesbianas, en aquellos momentos, fueron las primeras mujeres lesbianas que conocí. Y de ahí a la Asamblea de Mujeres de Bizkaia». Reflexionando en torno a la situación de las lesbianas, la entrevistada interpreta que su presencia era todavía bastante minoritaria y oculta dentro de un movimiento, el feminismo, cuya tarea estaba todavía centrada «en sacar un poco, en ponerle nombre a la lesbiana... ${ }^{10}$. Parte de

10. Entrevista a Isabel García. Nacida en Málaga en 1952, se trasladó en su adolescencia a Bilbao. Formó parte de la JOC, pasando posteriormente al MC y de ahí a LKI. Desde finales de los años setenta también formó parte de la Asamblea de Mujeres de Bizkaia y de colectivos de lesbianas.

Feminismo/s 33, junio 2019, pp. 199-223 
«Detrás de lo que quieren que seamos, está lo que somos». Revolución sexual y políticas sexuales feministas durante las décadas de los setenta y de los ochenta.

la invisibilidad del colectivo, en opinión de Pilar Ugalde, tenía que ver con el hecho que muchas de las lesbianas formaban parte de «grupos que se llamaban grupos de sexualidad, porque así, podían... no tenían que ser de lesbianas. Así, las que estaban ahí dentro podían decir: «No, no. Esto es un grupo de sexualidad. Las lesbianas son aquellas. Ahí solíamos tener bastante cristo...». Pese a la existencia de esas diferencias en cuanto al rol a desempeñar por el colectivo, ella rememora que se sintió gratamente sorprendida por encontrar «asambleas de mujeres, o coordinadoras feministas, que, de alguna manera, arropaban mucho a los colectivos de lesbianas» ${ }^{11}$.

Ugalde recuerda que al poco de tomar contacto con el movimiento feminista hizo su entrada «todo el tema del lesbianismo, que... hasta entonces había sido una enfermedad, que todas ocultábamos mucho... (y que) pasaba a ser otra cosa más a reivindicar». Estas palabras permiten adivinar una de las cuestiones centrales para comprender la importancia adquirida por el lesbianismo en el movimiento feminista, contemplado no tanto como una opción sexual, sino política, lo que resultaba en una posición identitaria muy sólida de «lesbiana feminista», comprometida y consciente. Su relato es muy significativo del empoderamiento experimentado por algunas lesbianas en el interior del movimiento de las mujeres. En un enclave de su memoria, la entrevistada insiste en evocar el descubrimiento de las políticas sexuales, en una reunión a la que acudió «con la excusa de que yo era militante de un partido, y era feminista. Y me acuerdo, según dijimos: «¡Esto no es una enfermedad! Y tal... ¡Esto es una cuestión política!, Porque el patriarcado...». ¡Ras! Me enganché ya a la cuestión política. Y ya dejé de ser una enferma». El cambio que refiere la entrevistada resulta también muy significativo de la exitosa interpelación conseguida por el discurso feminista, en el sentido de enlazar el pasado con el presente, y de configurar una posición política decididamente orientada hacia el futuro: «No sabes tú, o sea, para mí, lo que significó -refería Ugalde- ese lazo para agarrarme del feminismo, a aquello... a decir: «El patriarcado, lo mismo que nos ha condenado a las mujeres a no

11. Entrevista a Pilar Ugalde. Nacida en Rentería en 1958, formó parte de OIC y EMK, pasando a formar parte del movimiento feminista a finales de los años setenta. Militó en la Asamblea de Mujeres de Rentería, de colectivos de lesbianas feministas, y continúa en la actualidad trabajando en el movimiento, como integrante de un partido político, Plazandreok, compuesto exclusivamente por mujeres.

Feminismo/s 33, junio 2019, pp. 199-223 
«Detrás de lo que quieren que seamos, está lo que somos». Revolución sexual y políticas sexuales feministas durante las décadas de los setenta y de los ochenta. Una aproximación al caso del País Vasco

sé qué, ha condenado también esto...». Y para mí fue... o sea, la..., el ir contra la heterosexualidad como una manera de opresión, la heterosexualidad impuesta... aquello fue para mí la hostia» ${ }^{12}$.

Como puede inferirse de la documentación estudiada y en las palabras de esta entrevistada, el lesbianismo ya había sido investido desde finales de los setenta de un significado eminentemente político, al interpretarse como un elemento capaz de cohesionar y edificar una comunidad utópica movida por la sororidad y el amor compartido entre mujeres: «cuando te juntas con tus hermanas, ¿qué sucede? descubrimos otro mundo, empezamos a querernos a nosotras mismas un poco más y, a querer sobre todo a nuestras hermanas las mujeres, a gustarnos sus cuerpos, sus gestos, su dulzura, sus movimientos, a sentirnos cómplices, a descubrir en ellas y en nosotras un montón de posibilidades...», afirmaban. El movimiento se vinculaba así de modo indisoluble con la «atracción irresistible hacia otro mundo con otra escala de valores y sensaciones», esto es, con un significado utópico. «Descubrimos nuestros cuerpos y mezcladas nos reconocemos-concluía el texto- porque tu deseo es mi deseo, porque lo que sientes es lo que siento» (Jornadas de la Mujer de Euskadi, I: 16). La creación de colectivos específicos de lesbianas feministas a inicios de los años ochenta supuso un nuevo impulso para las políticas sexuales del feminismo, al relacionar el lesbianismo con la capacidad de subvertir en conjunto los fundamentos sociales, alegando que «a esta sociedad no le interesa en absoluto la existencia de unas mujeres que rompen con las normas porque se les desbarajusta todo el montaje. Las mujeres lesbianas concienciadas ponemos en peligro esta sociedad machista, patriarcal y capitalista» (Mujeres de EHGAM-Guipúzcoa 25). La práctica de lesbianismo, por tanto, era concebida como un verdadero salto cualitativo para el feminismo, pasando en muy poco tiempo de ser una cuestión «privada» a una reivindicación a asumir por el conjunto de las mujeres del movimiento:

Después de muchos siglos de un silencio total sobre la homosexualidad femenina parece ser que en los últimos tiempos ya se empiezan a oír leves rumores sobre la existencia de $<$ tal cosa $>$ nosotras, que somos personas con una sexualidad propia, y que además la nuestra dista mucho de esa ansia de dominación que conlleva el actual modelo de sexualidad masculina. Y lo

12. Entrevista a Pilar Ugalde.

Feminismo/s 33, junio 2019, pp. 199-223 
«Detrás de lo que quieren que seamos, está lo que somos». Revolución sexual y políticas sexuales feministas durante las décadas de los setenta y de los ochenta. Una aproximación al caso del País Vasco

hemos descubierto porque a hacer el amor se aprende haciéndolo, y también enseñando a hacerlo («Lesbianismo normalización» 21).

El movimiento feminista experimentó durante los años ochenta una fuerte evolución en torno a sus posturas sobre el lesbianismo. Su defensa, en opinión de un grupo de lesbianas feministas de Madrid publicado en la revista Zer Egin, no se relacionaba sólo ni básicamente (en) defender el derecho de las lesbianas a serlo», sino en «criticar las relaciones sexuales hoy dominantes». El lesbianismo, por tanto, no era concebido como una orientación sexual más o menos respetable (dando el respeto por hecho), sino como un gesto eminentemente político, propio de un grupo autodefinido como quienes se habían «atrevido a rebelarnos contra la hipócrita moral vigente que sostiene la gran mentira de que la sexualidad de la mujer encuentra su culminación en el hombre» («Romper la norma»13). Convertido en una pieza fundamental de la política sexual, el lesbianismo se interpretaba tanto como la máxima expresión de rebeldía y rechazo contra la norma heterosexual, así como la muestra más pura de feminismo y de lealtad a la transformación representada por el movimiento de las mujeres. La inclusión de las prácticas lesbianas en su programa sexual fue concebida como un salto para el movimiento, pasando en muy poco tiempo de ser una cuestión «privada» a hacerse extensiva, idealmente, al conjunto de mujeres que formaban parte de los grupos feministas.

En una entrevista concedida a la revista Sorginak en 1986, editada por colectivos de lesbianas del País Vasco, una activista identificada como «Miren», de veintisiete años de edad, aseguraba que la norma heterosexual era «tan fuerte y tan arraigada en la sociedad que ni siquiera las mujeres feministas estamos libres de ellas», razón por la cual había que realizar un doble esfuerzo, «por acercar a las mujeres lesbianas al MF y (por) hacer que el conjunto de mujeres del movimiento entiendan lo importante que es para la liberación de las mujeres rechazar la norma heterosexual como una pieza muy importante de la opresión patriarcal» («Esta es mi historia... y la tuya» 13). La incorporación de prácticas lesbianas, tal y como defendían las organizadoras del II Encuentro de Lesbianas Feministas de Orio en 1987, debía convertirse en el «tema fundamental de debate en las Asambleas de Donostia y Orereta y en las asambleas de Gipuzkoa, pero tema de debate de todas las mujeres, es decir, como debate del MF» («Sexualitate eta amatasun» 16). La estrategia de separación del feminismo lésbico representado por las

Feminismo/s 33, junio 2019, pp. 199-223 
«Detrás de lo que quieren que seamos, está lo que somos». Revolución sexual y políticas sexuales feministas durante las décadas de los setenta y de los ochenta. Una aproximación al caso del País Vasco

teorías de Gretel Amman o Rita Mae Brown ya había irrumpido con mucha fuerza en las II Jornadas Feministas del Estado español, celebradas en 1979 en la ciudad de Granada. Habría que esperar sin embargo a las jornadas de Orio para apreciar el apogeo de este tipo de planteamientos, afirmando que «el lesbianismo político es un concepto nuevo, un empuje, una connotación de radicalidad; no es solamente algo revulsivo, sino creador, que puede dar nuevos aires al movimiento feminista, es un elemento de análisis no acabado e incipiente, pero no por ello menos transformador y revolucionario» (Jornadas de Lesbianas Feministas de Euskadi 65).

Entre las múltiples propuestas de incorporación del lesbianismo como elemento definitorio y transformador de la conciencia y la praxis feminista, destacan las contenidas en un anexo titulado «Lesbianismo: ¿utopía?», que formó parte de los textos para el debate en las jornadas de Orio ${ }^{13}$. En dicho dosier, se incluía un texto con el título de «Crítica a las relaciones heterosexuales y significado del planteamiento de las relaciones lesbianas», que destaca por su novedad y claridad en los términos y su proximidad con algunos planteamientos separatistas del feminismo lésbico. El texto, firmado por Rita Mae Brown y publicado originalmente con el título «Le monde à venir», planteaba una enconada defensa de la práctica sexual lesbiana interpretada como la única vía capaz de desarrollar al máximo la potencialidad del feminismo. Así, el texto cargaba con dureza contra las mujeres que no se adaptaban en exclusiva a la práctica lesbiana, extendiendo sobre ellas una sombra de sospecha al entender que imposibilitaban llevar a cabo la política sexual propuesta desde el movimiento.

A cambio de fastidiar las comunidades de mujeres, a cambio de esta traición a las otras mujeres, obtienen efectivamente los privilegios de los hombres [...] Si eres una lesbiana visible, estás excluida de estos privilegios. Estás de una forma total contra la dominación del macho. ¿Cómo una mujer heterosexual ligada a los hombres, puede evitar traicionar a sus hermanas? [...] ¿Cómo vamos a construir un movimiento político serio si las mujeres nos

13. El anexo contaba de tres textos principales muy indicativos de distintas sensibilidades del movimiento insertas en el «separatismo lésbico»; uno de Monique Wittig titulado «No somos ni hombres ni mujeres», otro de Colette Guillaumin, de fuerte inspiración marxista, titulado «Critica a la sociedad heterosexual. La apropiación de las mujeres» y, por último, el trabajo de Mae Brown.

Feminismo/s 33, junio 2019, pp. 199-223 
«Detrás de lo que quieren que seamos, está lo que somos». Revolución sexual y políticas sexuales feministas durante las décadas de los setenta y de los ochenta. Una aproximación al caso del País Vasco

damos de golpes las unas contra las otras? No es posible. El lesbianismo es una etapa necesaria de la lucha por nuestra liberación [...] ¿Quiere decir esto que dejemos de lado a las heterosexuales? No. Pero no trabajaremos con nadie hasta que no hayan cambiado su comportamiento ( «Sexualitate eta amatasun» 31-32).

En un testimonio muy marcado por el tono crítico y de desmitificación, Edurne Eraso alude a los «dolores de cabeza», que tuvieron que ver con que se dio «un momento en el que el lesbianismo tuvo un auge, en el que el lesbianismo se empezó a ver como... como una posición política. No es que tú fueras... eh, feminista y lesbiana, sino que es que tú... tú eras lesbiana feminista, o sea, que estaba por delante, ¿no?». Reflexionando de modo retrospectivo sobre las ideas defendidas por una parte de sus compañeras, Eraso entiende que: «Ellas hacían algunas propuestas sobre la sexualidad y tal que a mí me costó, me costó... tuve mis momentos, luego volví a estar con mujeres... ahora salgo con un chico... luego volví a andar con mujeres. O sea, fue como... esos saltos, se hicieron difíciles porque... hay una influencia política también muy grande». Tras una prolongada trayectoria en distintos colectivos y espacios de mujeres durante la práctica totalidad del período abordado en este artículo, esta guipuzcoana considera que el peso de la identidad lesbiana en el movimiento fue un tanto excesivo, en el sentido de que «el movimiento de lesbianas aquí era tan rollo de...eres o no eres. O sea, la bisexualidad estaba muy mal vista, había un rechazo muy grande a las mujeres bisexuales» ${ }^{14}$.

A lo largo de este texto se han abordado de modo introductorio algunos de los principales aspectos de la revolución sexual y de las políticas sexuales propuestas por el feminismo durante las décadas de los años setenta y ochenta. Las distintas fuentes consultadas y/o construidas durante el transcurso de esta investigación apuntan a la importancia adquirida por la sexualidad en un sentido político, dando paso a una intensa producción de significados con respecto a este ámbito. Las políticas sexuales feministas no sólo comportaron profundas teorizaciones en torno al cuerpo y el sexo como campos de batalla de un movimiento emergente, sino que también comportaron toda una serie de propuestas concretas que, incorporadas por la subjetividad de las militantes, se orientaron a desestabilizar la sexualidad y la norma dominantes. Entre

14. Entrevista a Edurne Eraso (seudónimo).

Feminismo/s 33, junio 2019, pp. 199-223 
«Detrás de lo que quieren que seamos, está lo que somos». Revolución sexual y políticas sexuales feministas durante las décadas de los setenta y de los ochenta. Una aproximación al caso del País Vasco

estas prácticas nos hemos centrado en analizar la crítica al coito, la reivindicación del clítoris y el orgasmo femenino, o la asimilación de la práctica lesbiana. Todas estas cuestiones debatidas por las mujeres sirvieron para cohesionar el movimiento feminista a lo largo de las décadas de los años setenta y ochenta, aunque generaron algunos disensos por lo que algunas interpretaron como una cierta rigidez en algunos planteamientos. Sin embargo, este tipo de disensos no acabaron con el movimiento, sino que lo enriquecieron mediante una búsqueda incesante de nuevas perspectivas y posiciones políticas que sin duda exceden los límites de este texto y se extienden hasta la actualidad, invitando como lo hicieron las mujeres de los setenta a repensar la sexualidad desde prismas distintos al del varón heterosexual y machista.

\section{REFERENCIAS BIBLIOGRÁFICAS}

Angulo, Javier. «Las feministas, por la libertad sexual». El País 13 diciembre 1979. Disponible también en Jornadas de la Mujer de Euskadi, 8-11 diciembre 1977: 13.

Beorlegui, David. «Expectativas de género en las luchas obreras del tardofranquismo y la transición. Feminismo y Memoria en el Gran Bilbao (19751979)». Historia Social 88 (2017): 47-62.

Blasco, Inmaculada. «Definir y explicar el feminismo histórico. Debates y proyectos de análisis desde la historia». Feminismos. Contribuciones desde la historia. Eds. Ángela Cenarro y Régine Illon. Zaragoza: Prensas Universitarias de Zaragoza, 2014. 267-289.

Comisión antiagresión de la AMB. «Sobre la violación». Egin 6 marzo 1983: 16. Epelde, Edurne, Miren Aranguren e Iratxe Retolaza. Gure genealogía feminista. Tafalla: Txalaparta, 2015.

Esteban, Mari Luz. «Cuerpos políticos feministas. El feminismo como cuerpo». Cuerpos políticos y agencia. Reflexiones sobre cuerpo, trabajo y colonialidad. Coords. Cristina Villalva y Nacho Álvarez. Granada: Universidad de Granada, 2011. 45-84.

Garaizábal, Cristina. «Política sexual feminista», ponencia presentada en las Jornadas Feministas de Granada en 2009, p. 2. http://www.aldarte.org/comun/ imagenes/documentos/Debates_feministas_sobre_la_sexualidad-_Cristina_ Garaizabal.pdf. 28-06-2018.

Feminismo/s 33, junio 2019, pp. 199-223 
«Detrás de lo que quieren que seamos, está lo que somos». Revolución sexual y políticas sexuales feministas durante las décadas de los setenta y de los ochenta. Una aproximación al caso del País Vasco

Hooks, Bell. El feminismo es para todo el mundo. Madrid: Traficantes de Sueños, 2017.

Jornadas de la Mujer de Euskadi, 8-11 diciembre 1977. Disponible en el Centro de Documentación de las Mujeres Maite Albiz.

Larumbe, María Ángeles. Las que dijeron que no. Palabra y acción del feminismo en la Transición. Zaragoza: Prensas Universitarias de Zaragoza, 2004.

«Lesbianismo normalización». Egin 5 abril 1983: 21.

Llona, Miren. Entreverse. Teoría y metodología practica de las fuentes orales. Bilbao: Universidad del País Vasco/ Euskal Herriko Unibertsitatea, 2012.

Martínez, Carmen, Purificación Gutiérrez, y Pilar González, eds. El movimiento feminista en España en los años 70. Madrid: Fundación Pablo Iglesias, Ediciones Cátedra, Universitat de València, 2009.

Miren,»Esta es mi historia... y la tuya», Sorginak 1, febrero 1986: 11-13

Mujeres de EHGAM-Guipúzcoa. «Es muy duro ir de lesbiana por la vida». Egin 19 junio 1983: 25.

Mujeres jóvenes de Institutos, FP Y Facultades, «Basta», Geu Emakumeok 3, 27 junio 1985: 3

Nash, Mary. Mujeres en el mundo. Historia, retos y movimientos. Madrid: Alianza, 2012.

«Romper la norma». Zer Egin 23 de junio-7 de julio 1984: 13.

«Sobre el amor romántico y la sexualidad», Geu Emakumeok 3, 27 junio 1985: 2 Rubin, Gayle. «Thinking Sex: Notes for a Radical Theory of the Politics of Sexuality». Pleasure and Danger. Ed. S. Carole Vance. London/Boston: Routledge-Kegan, 1984: 267-320.

II Jornadas de Lesbianas Feministas de Euskadi, Orio, 1-3 mayo de 1987. Disponible en el Centro de Documentación de las Mujeres Maite Albiz.

«Sexualitate eta amatasun askatu baten alde. Por una sexualidad y una maternidad libres», dosier de la Comisión de la Mujer de Algorta, 1981.

Uría, Paloma. El feminismo que no llegó al poder. Trayectoria de un feminismo crítico. Madrid: Talasa, 2009.

Willis, Ellen. No more nice girls. Countercultural essays. Hanover: Wesleyan University Press, 1992.

Zabala, Begoña. Movimiento de mujeres, mujeres en movimiento. Tafalla: Txalaparta, 2008. 